\title{
Letter and Colour Matching Tasks: Parametric Measures of Developmental Working Memory Capacity
}

\author{
Tamara L. Powell, ${ }^{1,2}$ Marie Arsalidou, ${ }^{1,3,4}$ Vanessa M. Vogan, ${ }^{1,5}$ and Margot J. Taylor ${ }^{1,6}$ \\ ${ }^{1}$ Department of Diagnostic Imaging and Neurosciences and Mental Health Programme, Research Institute, Hospital for Sick Children, \\ University of Toronto, 555 University Avenue, Toronto, ON, Canada M5G 1X8 \\ ${ }^{2}$ Department of Psychology and Language Sciences, University College London, 26 Bedford Way, London WC1H 0AP, UK \\ ${ }^{3}$ Department of Psychology, National Research University Higher School of Economics, Myasnitskaya Street 20, Moscow 101000, Russia \\ ${ }^{4}$ Department of Psychology, Faculty of Health, York University, 4700 Keele Street, Toronto, ON, Canada M3J 1P3 \\ ${ }^{5}$ Applied Psychology and Human Development, Ontario Institute for Studies in Education, University of Toronto, 252 Bloor Street W., \\ Toronto, ON, Canada M5S 1V6 \\ ${ }^{6}$ Department of Psychology, University of Toronto, 27 King's College Circle, Toronto, ON, Canada M5S 1A1
}

Correspondence should be addressed to Tamara L. Powell; tamarapowell6@gmail.com

Received 22 July 2014; Revised 9 November 2014; Accepted 11 November 2014; Published 30 November 2014

Academic Editor: Andrew N. Meltzoff

Copyright (C) 2014 Tamara L. Powell et al. This is an open access article distributed under the Creative Commons Attribution License, which permits unrestricted use, distribution, and reproduction in any medium, provided the original work is properly cited.

We investigated the mediating role of interference in developmental assessments of working memory (WM) capacity across childhood, adolescence, and young adulthood. One hundred and forty-two participants completed two versions of visuospatial (colour matching task, CMT) and verbal (letter matching task, LMT) WM tasks, which systematically varied cognitive load in a high and low interference condition. Results showed similar developmental trajectories across high interference contexts (CMTand LMT-Complex) and divergent developmental growth patterns across low interference contexts (CMT- and LMT-Simple). Performance on tasks requiring greater cognitive control was in closer agreement with developmental predictions relative to simple recall guided tasks that rely solely on the storage components of WM. These findings suggest that developmental WM capacity, as measured by the CMT and LMT paradigms, can be better quantified using high interference contexts, in both content domains, and demonstrate steady increases in WM through to mid-adolescence.

\section{Introduction}

Working memory (WM) refers to a cognitive system that temporarily maintains and manipulates information, which is crucial for performance on a wide range of cognitive tasks. WM plays an important role in reasoning and learning [1], language comprehension [2], and executive function $[3,4]$ and is a central component of intelligent behaviour [5]. Despite considerable research devoted to examining individual differences in WM, there is not yet any consensus in the literature as to the storage limits or developmental trajectories expected in either the visual or the verbal WM domain [6]. Discrepancies in developmental estimates may be due, in part, to inconsistencies in ways of operationally defining, assessing, and measuring cognitive demand in tasks of varying complexity [7]. The present study addresses this issue by highlighting the mediating role controlled interference plays in the measurement of WM across development.

A main challenge in extracting a general trajectory of WM across development has been the limitations of narrow age ranges and the relative absence of adolescents and young adults within the developmental literature. A great deal of the behavioural research on WM has come from studies of children in their preschool or early school-age years (e.g., [810]), but less in later childhood. This is unfortunate given the increasing evidence for protracted development of the frontal lobes and the functions they support well into adulthood [11]. Although some recent studies have explored this 
prolonged maturation and its association with improvements in behavioural performance on WM tasks, inconsistencies in testing methods have yielded inconclusive results (for a review, see [12]). The findings are further complicated by methodological variability such as prior learning, effect, and rehearsal strategies.

Most developmental theorists agree that WM capacity increases as children mature; however, the precise rate and age at which these abilities develop and are fully mature remain a lively debate. For instance, Halford and colleagues [16] posit that storage capacity rises in tandem with reasoning ability, reaching WM limits of 1, 2, 3, and 4 at ages 1, 1.5, 5, and 11 year olds, respectively. However, other developmental theorists postulate that WM increases by one informational unit every other year, from one unit at the age of three to the adult capacity of seven units at the age of 15 [1315]. The former model [16] is concordant with Cowan's [17] adult central WM capacity limit of four chunks, whereas the latter [14] is in line with Miller's [18] classic "magical" number 7 capacity. Discrepancies in the rate of change for developmental WM can be attributed to a number of factors including, but not limited to, how performance is measured, the response modality, the strength of the response bias, and the degree of simultaneous WM demands imposed by the task [7]. Differences in WM capacity limits, however, can be accounted by the level of imposed control on processing strategies (e.g., verbal rehearsal or grouping of items together) and sensory specific information (e.g., how familiar a picture looks or voice sounds in the stimulus presentation) [19]. Such methodological issues have limited the consistency of results across studies and the establishment of growth patterns that characterize normative development of WM. In moving forward, a general developmental trajectory adds value to the existing central capacity theorem that focuses primarily on developmental differences (e.g., between adults and children) [17] rather than developmental growth patterns across time. This framework will additionally offer an evidence base to corroborate or refute the existence of a central capacity limit within both content modalities [16].

Many developmental theories to date have failed to adequately account for cross-task variation in the estimation of WM capacity [6]. With this in mind, we employed an explicit computational measure of developmental WM capacity that considers situational features and procedural and figurative sources in its quantification. Process task analysis is a rationally based method used to estimate task difficulty on a single common metric (e.g., the number of WM capacity units needed to solve a task) $[13,15,20]$. The degree of cognitive control required to complete a task is proportionally weighted in the Metasubjective Task Analysis (Appendix A in Supplementary Material available online at http://dx.doi.org/10.1155/2014/961781) computation of individual WM scores, such that tasks requiring greater cognitive control are accredited additional WM unit(s). It can therefore be used across paradigms of varying complexity, such as our simple and complex CMT and LMT tasks.

Analytical approaches to measuring cognitive constructs, such as WM, have been gaining momentum in recent years, and there have been a number of cross-sectional reports that have substantiated the predicted WM trajectory employed in the present study $[13,15,21,22]$. Using varied cognitive loads and consistent executive demands, such studies have yielded reliable estimates of developmental growth that show that children aged 7-8, 9-10,11-12, 13-14, and 15 years and older have a WM capacity of 3, 4, 5, 6, and 7 items, respectively. This WM trajectory appears to remain relatively invariant across WM domain (e.g., visuospatial and verbal) [23], culture [24], and socioeconomic class [25], leading many researchers to infer that this ability develops endogenously as a result of biological or epigenetic factors $[22,26]$. As such, developmental WM is expected to develop in each respective content domain in conjunction with neurodevelopmental changes in areas known to support WM neural circuitry. Understanding the development of WM capacity in normative populations may serve as a benchmark for identifying cognitive impairments in clinical populations.

Complex tasks can be contrasted with simple tasks in that they comprise two different modes of selective attention [27]. For instance, automatic mental attention, defined as effortless cue-driven attention activated by salient or novel experiences, is typically only evoked under simple contexts [26], that is, situations that only activate relevant responses for the task at hand. Voluntary mental attention, on the other hand, requires active, executive-driven attending, elicited through complex tasks $[26,28]$. Practice and or familiarity acquired through past experiences or learning can impede the ability to distinguish task complexity [29], as maturation and learning are complementary processes that show a dialectical interaction (e.g., intertwining, trade-off relations) throughout development $[15,24]$. It is for this reason that we attempted to minimize prior learning and rehearsal in measuring WM processing since both casual constructs can obscure the clear observation of each other, if studied within the same paradigm or situation.

In the current study we used visuospatial and verbal WM tasks that systematically varied cognitive load in two conditions, which were defined in terms of complexity. The level of interference differentiating tasks of lower and higher complexity was delineated in an analysis of task processing demand (see Appendix A) and in turn converted to units of measurement for the final computation of subjects' WM capacity. Our aim in employing this process task analysis was to measure reliably whether performance on WM tasks, particularly tasks eliciting executive control [30] and active maintenance strategies, improves with age in parallel with known brain maturational events. The established link between interference and WM capacity in behavioural [31] and neuroimaging $[32,33]$ research underscores the need to examine behavioural performance in a large developmental sample using comparable paradigms that only differ in context and domain. Our study addressed this issue by investigating WM at different stages of development, specifically from the onset of spontaneous verbal rehearsal strategies at the age of 7 (see [34], for a review) to higher order cognitive processes in adolescence and early adulthood [35].

A number of previous studies have shown stable developmental changes in WM which appear only within complex tasks, across WM domains [13, 15, 23, 36]. In fact, 
process-specific models of WM suggest distinct functional circuits which subserve processing-related versus storagespecific tasks [37]. Vogan et al. [38] used the current complex visuospatial paradigm to examine differences in WM load and neural activations in a clinical and normative sample of children aged 7 to 13 years and found typically that developing children rely mainly on areas within the frontal and parietal lobes-critical substrates for WM. Both this study and its immediate precursor [13] demonstrated a stagewise progression of WM in children from late childhood to early adolescence. We accordingly hypothesized that complex tasks would better predict maturational growth of visuospatial and verbal WM across development relative to simple tasks, which evoke low task interference. Further, we predicted that performance in high interference tasks would be relatively invariant across visuospatial and verbal domains for each age group. Finally, simple tasks with fewer cognitive constraints were expected to yield less reliable estimates of developmental growth and thus diverge from the theoretical predictions.

\section{Materials and Methods}

2.1. Participants. We tested participants in the following six age groups: children aged 7-8 $(n=23, M=7.83), 9-10$ $(n=25, M=9.78), 11-12(n=21, M=11.84), 13-14$ $(n=22, M=13.74)$, and $15-16(n=25, M=15.57)$ years and young adults $(n=26, M=24.74$, range: $20-$ 32 years), for a total of 142 subjects, 86 male and 56 female. Participants were recruited from the community (adults) and three urban Toronto public schools in culturally diverse middle-income neighborhoods. Children were enrolled in mainstream classes and teachers confirmed that students included in the study did not have a history of learning disability, dyslexia, or any other language impairment. All families signed an informed consent agreeing to participate in the study, and the Research Ethics Board at the Hospital for Sick Children approved all procedures.

2.2. Materials. Two paradigms were run with all participants: a colour matching task (CMT) and a letter matching task (LMT). The CMT is a validated visuospatial WM capacity task [13] and the LMT is a newly developed verbal analogue of the CMT (Figure 1). Each simple task (CMT-Simple, LMT-Simple) had a corresponding complex version (CMTComplex, LMT-Complex) that contained task-relevant and task-irrelevant (to be ignored) items. Complex tasks were differentiated from simple tasks through the inclusion of embedded invalid cues (e.g., distractors), which required individuals to inhibit impulsive responses and ignore aspects of the stimuli. Irrelevant task colours and letters were ignored across conditions to ensure that baseline levels of executive function were adequately controlled in all tasks. These items also served as a background for creating a frame in which the relevant stimuli would be presented.

The $n$-back task is commonly used to assess graded WM capacity (see [39] for a review). This protocol requires participants to view a sequence of stimuli and match the current stimulus with one $n$ (e.g., $0,1,2$, or 3) step earlier in the sequence. The load factor $n$ can be adjusted to manipulate task difficulty level. Increases in task difficulty (e.g., WM load), however, also increases the number interfering stimuli between the target and criterion set, thus requiring subjects to exercise a number of different mental strategies in finding a task solution (e.g., 0-back, recognition; 1-back, maintenance; 2-back, maintenance and monitoring). The CMT and LMT paradigm is advantageous in that it systematically manipulates WM load while keeping executive function (i.e., number and kind of operations needed to solve a task) constant across all difficulty levels. The only changing demand in our novel paradigms is the number of relevant items (e.g., letters or colours) to keep in mind.

In the LMT, the Square task was considered simple as letters were presented on a uniformly spaced 4 by 3 square, thus facilitating recognition of relevant items. In the complex task, however, items were presented on a large figural A letter (Figure 1); the use of a figural A was intended to elicit inhibitory control that required participants to ignore "A" as a letter while processing the relevant test items. In both tasks the letters "O" and "P" were irrelevant letters and also to be ignored.

For the CMT, the Balloon task was the simple versionas the set of balloons was a collection of discrete shapes that were relatively uniform in shape and size. Colour was the only variant between figures. In contrast, the more difficult Clown task presents a complex condition, as participants were required to ignore the clown's face and, as with the figural A, the holistic of the clown in general. The colours blue and green in both CMT tasks were irrelevant and also were to be ignored (Figure 1).

Using a 1-back protocol, participants were asked to indicate whether the relevant features (colours or letters) in each stimulus matched those presented in the previous stimulus, disregarding location and repetition of items (letters or colours). Participants had three simultaneous considerations (1) to identify relevant letters/colours within the figure; (2) to determine whether it contained the same or different letters/colours as the preceding figure; and (3) to do so as fast and as accurately as possible. Matching responses presented $50 \%$ of the time across all tasks, and when successive figures differed, task-relevant items varied by one ( $92 \%$ of changes) or two ( $8 \%$ of changes) colours or letters.

One item was added to each task for each difficulty level, producing a steady, parametric increase in demand on WM capacity while not changing the executive function aspects of the task (i.e., across difficulty levels, it remained a 1back task, with WM maintenance strategies and delays are kept constant). Each addition of an item resulted in a WM demand of $n+1$, where $n$ represents the total number of relevant colours or letters (e.g., difficulty level $[D] ; n=1-$ $6)$ presented. The equivalents of two items $(n+2)$ were added to each complex task, which required participants to process embedded stimuli from perceptually salient figures (clown and figural A), as additional cognitive resources are needed to mitigate the effects of contextual influences. For all tasks, WM scores represent the highest number of items $(n=2-$ 8) a participant passed reliably, with at least $70 \%$ accuracy. For example, a participant with an accuracy score of $85 \%$ 

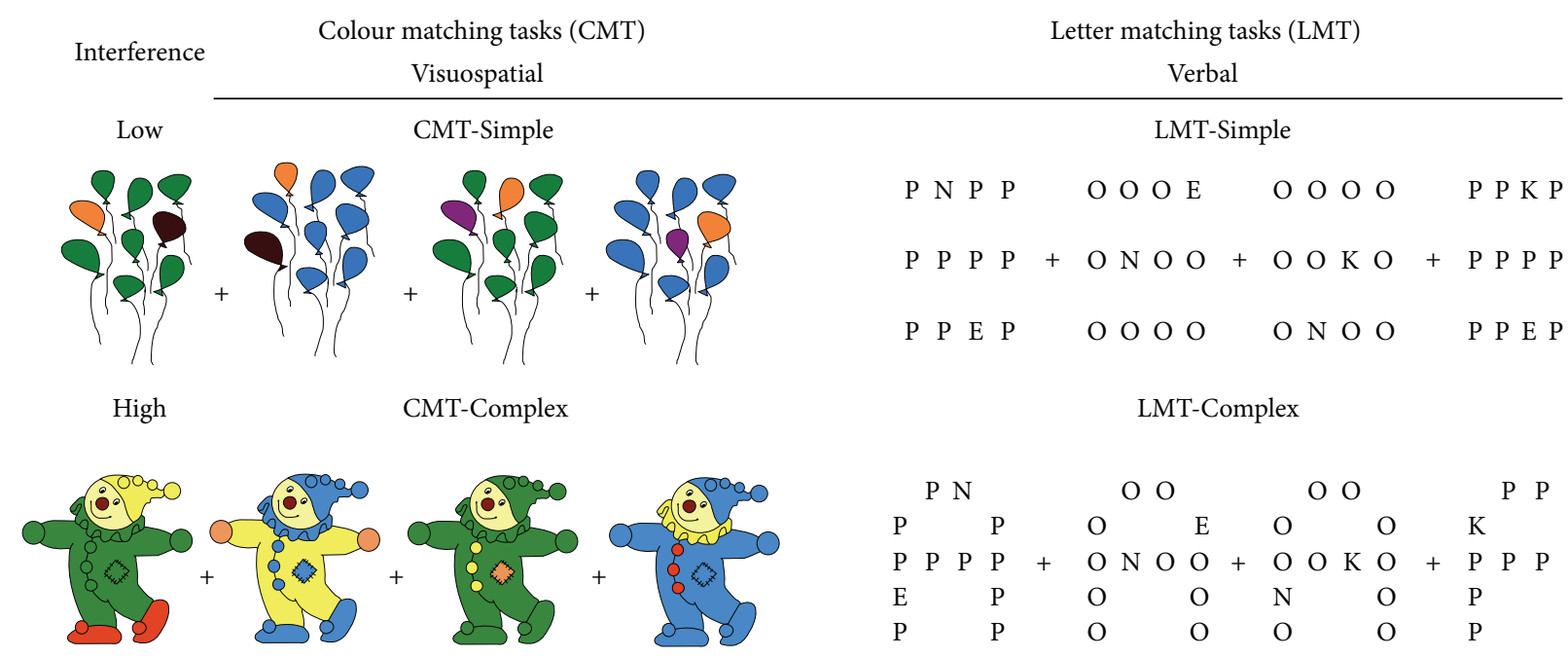

\begin{tabular}{|c|c|c|c|c|c|c|c|c|c|c|c|c|}
\hline \multicolumn{2}{|l|}{$\mathrm{P} N$} & & \multicolumn{3}{|c|}{$\mathrm{OO}$} & \multicolumn{3}{|c|}{$\mathrm{OO}$} & & \multicolumn{3}{|c|}{$\mathrm{P} P$} \\
\hline P & $\mathrm{P}$ & & $\mathrm{O}$ & E & & $\mathrm{O}$ & & $\mathrm{O}$ & & K & & \\
\hline $\mathrm{P} \quad \mathrm{P} \quad \mathrm{P}$ & $\mathrm{P}$ & + & $\mathrm{O} \mathrm{N}$ & $\mathrm{O} \mathrm{O}$ & + & $\mathrm{OC}$ & $\mathrm{OK}$ & $\mathrm{O}$ & + & $\mathrm{P}$ & & $\mathrm{P}$ \\
\hline E & $\mathrm{P}$ & & $\mathrm{O}$ & $\mathrm{O}$ & & $\mathrm{N}$ & & $\mathrm{O}$ & & $\mathrm{P}$ & & \\
\hline $\mathrm{P}$ & $\mathrm{P}$ & & $\mathrm{O}$ & $\mathrm{O}$ & & $\mathrm{O}$ & & $\mathrm{O}$ & & P & & \\
\hline
\end{tabular}

Figure 1: The colour matching tasks (CMT) and letter matching tasks (LMT). Simple and complex figures in the CMT (left) and LMT (right) tasks. A WM capacity of 3 (WM capacity $=(\#$ of colours/letters $)+1)$ is shown for the simple CMT and LMT (top), and a WM capacity of $4(\mathrm{WM}$ capacity $=(\#$ of colours/letters $)+2)$ for the complex (bottom) CMT and LMT tasks. In both versions children are taught to ignore irrelevant colours (blue and green) and letters ("O" and "P") and the location and repetition of test items. The complex versions also require participants to ignore the clown's face and the large figural A, in the CMT and LMT, respectively.

(D2), 79\% (D3), 75\% (D4), 71\% (D5), 68\% (D6), 62\% (D7), and $58 \%(D 8)$ would pass difficulty level 5 (relevant letters $[n]=3+2$ ), consequently exhibiting a WM capacity of 5 . Trials were scored as incorrect if participants did not respond within $3 \mathrm{~s}$ of the stimulus presentation or if they responded incorrectly.

2.3. Procedure. Participants completed either the CMT or the LMT on two different sessions within a 2-week period of time. Training was given prior to each session, during which participants were shown the respective WM task and instructed to ignore irrelevant stimuli (e.g., blue and green; "O" and "P") and distractors (e.g., clown's face and larger letter $\mathrm{A}$ ), while focusing on relevant test items in the CMT (yellow, purple, pink, red, orange, brown, and grey) and in the LMT (A, B, E, H, K, M, and N). A 12-trial computerized training session was provided with feedback to ensure that participants were familiar with the task and instructions. Task figures were presented one at a time, in sequence, and participants responded by using a standard keyboard pressing $(\cdot)$ for same and $(/)$ for different response choices, using left and right fore-fingers; the assignment of these response keys was counterbalanced across subjects.

Stimuli were shown for $3 \mathrm{~s}$ with an interstimulus interval of $1 \mathrm{~s}$ in both the training and the experimental sessions (Figure 2). A total of 24 blocks (192 stimuli) with 168 tasks and 24 baseline figures were presented across two successive runs. Each run consisted of twelve $32 \mathrm{~s}$ blocks, each containing eight stimuli. All six difficulty levels were presented in pseudorandom order and interleaved by $20 \mathrm{~s}$ rest blocks (baseline figures containing only task-irrelevant stimuli). Task order was counterbalanced across participants and response time and accuracy were recorded using Software Presentation (v. 10.1.109.26.06 Neurobehavioral Systems Inc.).

2.4. Data Screening and Analyses. Analyses were performed using SPSS software, version 20 (SPSS Inc., Chicago, IL). All data were reviewed for missing values, accuracy of data entry, and outliers prior to statistical analysis. $Z$-scores and scatterplots were used to identify outliers, classified as scores $3 \mathrm{SDs}$ from the mean. WM scores for each age group on all WM capacity tasks fell within 3 SDs of the mean, and with the exception of one grade 2 child who only completed the visuospatial tasks, data were obtained across all four WM capacity tasks for each participant.

Correlations between simple and complex visuospatial and verbal WM tasks are shown in Table 1. As expected, all four tasks were significantly correlated with each other as well as with chronological age, even after the variance explained by age was removed ( $p<0.01$, two-tailed). There were only marginal differences in the strength of correlations across domains and level of interference, with simple tasks accounting for $48 \%$ and complex $50 \%$ of the shared variance in WM scores. Similarly, visuospatial and verbal WM tasks demonstrated a shared variance of $58 \%$ and $67 \%$, respectively.

\section{Results}

WM scores for the CMT and LMT tasks were analysed using a 6 (age) $\times 4$ (task) repeated measures ANOVA. Mauchly's test indicated that the assumption of sphericity was violated $(\chi=21.55, p<0.01)$; therefore degrees of freedom were corrected using Huynh-Feldt $(\varepsilon=0.96)$. Results showed a significant effect of task $\left(F_{(2.87,387.67)}=22.28, p<0.001\right.$, partial $\left.\eta^{2}=0.14\right)$ and an effect of age $\left(_{(5,135)}=88.48\right.$, 


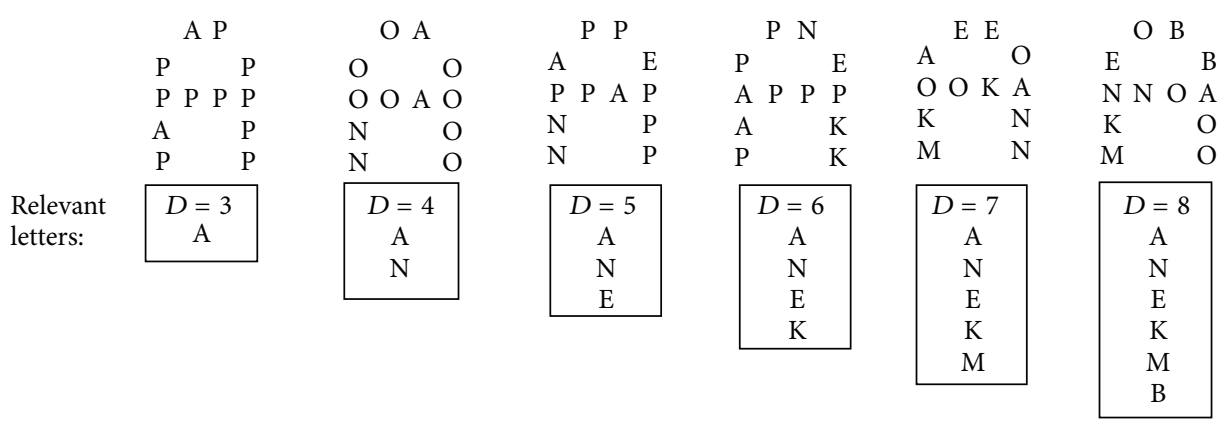

(a)
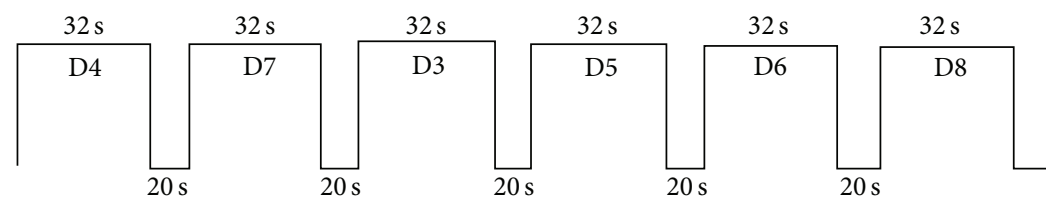

(b)

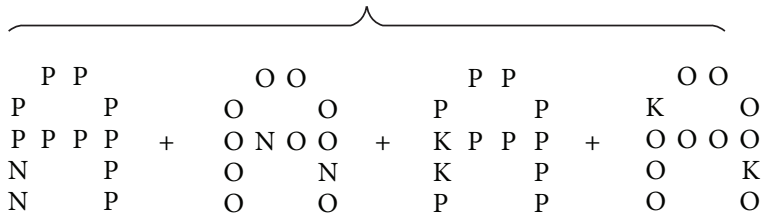

(d)

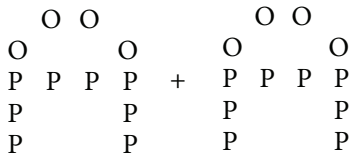

(c)

Figure 2: Protocol description for complex letter matching task (LMT). (a) There were six levels of increasing difficulty. The addition of one relevant letter $(\mathrm{A}, \mathrm{B}, \mathrm{E}, \mathrm{H}, \mathrm{K}, \mathrm{M}$, and $\mathrm{N}$ ) increased each difficulty level by one, such that difficulty = (\# letters +2$)$. (b) Within this block design task, each of the 2 runs contained six $32 \mathrm{~s}$ task blocks that were pseudorandomly ordered. (c) Each task block is immediately followed by a $20 \mathrm{~s}$ baseline block containing irrelevant letters $(\mathrm{O}$ and $\mathrm{P})$ to be ignored. (d) Example of four sequential figures in the task block for difficulty level 3. Stimuli are shown for $3 \mathrm{~s}$ and interleaved by a $1 \mathrm{~s}$ interstimulus cross.

TABLE 1: Correlations among WM task and age.

\begin{tabular}{lcccc}
\hline & CMT-S & CMT-C & LMT-S & LMT-C \\
\hline Age & $0.63^{* *}$ & $0.69^{* *}$ & $0.66^{* *}$ & $0.68^{* *}$ \\
CMT-S & - & $0.76^{* *}$ & $0.69^{* *}$ & $0.66^{* *}$ \\
CMT-C & $0.58^{* *}$ & - & $0.77^{* *}$ & $0.71^{* *}$ \\
LMT-S & $0.47^{* *}$ & $0.59^{* *}$ & - & $0.82^{* *}$ \\
LMT-C & $0.42^{* *}$ & $0.45^{* *}$ & $0.66^{* *}$ & - \\
\hline
\end{tabular}

Note. ${ }^{* *} p<0.01$ (2-tailed). Bivariate Pearson's $r$ correlations are above the diagonal and partial Pearson's $r$ correlations controlling for age are below the diagonal.

$p<0.001$, partial $\left.\eta^{2}=0.77\right)$. Post hoc tests of age, using Scheffe corrections, show a positive linear trend in performance with older participants outperforming younger participants across WM tasks (MD ranged from 1.07 to 4.15, $p<0.01$ ), except between groups of 11-12 years compared to $13-14$ years $(\mathrm{MD}=-0.46, p=0.65)$, as well as 15 to 16 years and young adults ( $\mathrm{MD}=-0.31, p=0.87$; see Figure 3 ).

On average, participant's WM scores were higher in the visuospatial (CMT-Simple, $M=5.42, \mathrm{SD}=1.78$;
CMT-Complex, $M=5.27, \mathrm{SD}=1.92)$ than the verbal WM tasks (LMT-Simple $M=4.61, \mathrm{SD}=1.83$; LMTComplex $M=4.92$, SD = 1.95), and as illustrated in Table 2, trajectories within these content domains varied with complexity. Interestingly, participants' WM scores appeared to be relatively invariant across complex tasks but differed significantly across most age groups in simple tasks (Table 2), thus driving an age $\times$ task interaction $\left(F_{(14.36,387.67)}=3.12\right.$, $p<0.001$, partial $\left.\eta^{2}=0.10\right)$.

Equivalence tests using paired sampling $t$-tests between obtained and predicted WM scores are shown in Table 3. Both CMT- and LMT-Complex scores agreed with theoretically predicted values of WM capacity across development. Consistent with previous research [13], CMT-Complex showed sustained equivalence across all age groups. Similarly, equivalence was shown in LMT-Complex for young adults and children aged 7-8, 11-12, and 13-14 years. On the LMT-Simple, however, students underperformed relative to theoretical predictions and their performance on other WM measures. Rapid improvements in WM scores that plateaued comparatively early in development characterized growth patterns in CMT-Simple. These patterns are consistent with short-term 
TABLE 2: Pair sample $t$-tests of WM scores across grade and WM capacity task.

\begin{tabular}{|c|c|c|c|c|}
\hline$t$-test & $\begin{array}{c}\text { Age group } \\
\text { (years) }\end{array}$ & CMT-Simple & & CMT-Complex \\
\hline \multirow{7}{*}{ Visuospatial } & 7-8 & 2.43 & $\neq$ & 2.83 \\
\hline & $9-10$ & 4.92 & $\neq$ & 4.12 \\
\hline & $11-12$ & 5.38 & $=$ & 4.86 \\
\hline & $13-14$ & 6.05 & $=$ & 5.50 \\
\hline & $15-16$ & 6.64 & $=$ & 6.80 \\
\hline & $18+$ & 6.88 & $=$ & 7.19 \\
\hline & & LMT-Simple & & LMT-Complex \\
\hline \multirow{7}{*}{ Verbal } & $7-8$ & 2.36 & $=$ & 2.77 \\
\hline & $9-10$ & 3.16 & $=$ & 3.24 \\
\hline & $11-12$ & 4.86 & $=$ & 4.67 \\
\hline & $13-14$ & 4.73 & $\neq$ & 5.32 \\
\hline & $15-16$ & 5.96 & $\neq$ & 6.48 \\
\hline & $18+$ & 6.31 & $=$ & 6.73 \\
\hline & & CMT-Simple & & LMT-Simple \\
\hline \multirow{7}{*}{ Simple } & $7-8$ & 2.50 & $=$ & 2.36 \\
\hline & $9-10$ & 4.92 & $\neq$ & 3.16 \\
\hline & $11-12$ & 5.38 & $=$ & 4.86 \\
\hline & $13-14$ & 6.05 & $\neq$ & 4.73 \\
\hline & $15-16$ & 6.64 & $\neq$ & 5.96 \\
\hline & $18+$ & 6.88 & $\neq$ & 6.31 \\
\hline & & CMT-Complex & & LMT-Complex \\
\hline \multirow{6}{*}{ Complex } & $7-8$ & 2.86 & $=$ & 2.77 \\
\hline & $9-10$ & 4.12 & $\neq$ & 3.24 \\
\hline & $11-12$ & 4.86 & $=$ & 4.67 \\
\hline & $13-14$ & 5.50 & $=$ & 5.32 \\
\hline & $15-16$ & 6.80 & $=$ & 6.48 \\
\hline & $18+$ & 7.19 & $=$ & 6.73 \\
\hline
\end{tabular}

Note. $p=0.05$. Equivalence tests were computed using paired sample $t$-tests and pairwise exclusions; =: equivalent; $\neq$ : not equivalent.

storage (e.g., basic ability to store target items independent of concurrent processing) models of WM assessing immediate serial recall [9].

\section{Discussion}

Our investigation of developmental WM capacity within the visuospatial and verbal WM domains showed that the ability to maintain and manipulate task-relevant information in active WM is best measured under conditions of high interference. Age-related changes in complex WM tasks increased linearly from three items at the age of seven to asymptote levels of performance of seven items at the age of 15 . Gradual improvement, evident by an increasing efficiency to override proponent responses, was observed through adolescence and into young adulthood.



FIGURE 3: Mean WM capacity scores as a function of age. Error bars represent standard error and red bars represent predicted WM capacity scores for each age group.

4.1. Visuospatial and Verbal WM. All age groups tended to perform better on the visuospatial than the verbal WM tasks, even though both were designed to employ similar cognitive processes (maintenance and manipulation) and attentional resources (e.g., executive control system). Differences in performance across content modalities, at least earlier in development, may be due in part to a heavier reliance on visual codes for active maintenance processes in childhood. Visual information is thought to require recoding into phonological form; however active rehearsal strategies that facilitate verbal recoding do not develop until late childhood [40]. Further, the age at which children begin to use an active rehearsal strategy may depend on the modality in which the stimuli are presented [41]. For young children, processing of visual and verbal information may occur in separate memory stores as shown by separate factor analysis loading of visual and verbal subtests from the Wide Range Assessment of Memory and Learning battery [42]. After 9 years of age however, visual memory subtests have similar loadings on the visuospatial and a verbal WM factor, suggesting the emergence of verbal recoding, which is used in concert with visual processes to boost WM performance [43]. Accordingly, the variability observed in WM capacity scores across complex visuospatial and verbal WM tasks in children aged 9-10 years, namely, lower scores within the LMT-Complex, may reflect maturational changes in verbal WM, specifically protracted development of verbal rehearsal strategies which are dependent on speech production and the rate at which it is processed (e.g., time required to internally rehearse a set of items; [44]). This explanation may also account for the lower WM scores 
TABLE 3: Equivalence tests among WM scores.

\begin{tabular}{|c|c|c|c|c|c|c|c|}
\hline Age group (years) & $t$-test & CMT-Simple & & WMp & & CMT-Complex & $t$-test \\
\hline $7-8$ & -2.19 & $2.43(1.24)$ & $\neq$ & 3 & $=$ & $2.83(0.94)$ & ns \\
\hline $9-10$ & 3.07 & $4.92(1.50)$ & $\neq$ & 4 & $=$ & $4.12(1.27)$ & ns \\
\hline $11-12$ & ns & $5.38(1.16)$ & $=$ & 5 & $=$ & $4.86(1.28)$ & ns \\
\hline $13-14$ & ns & $6.05(0.65)$ & $=$ & 6 & $=$ & $5.50(1.77)$ & ns \\
\hline $15-16$ & -3.67 & $6.64(0.49)$ & $\neq$ & 7 & $=$ & $6.80(0.82)$ & ns \\
\hline $18+$ & ns & $6.88(0.33)$ & $=$ & 7 & $=$ & $7.19(0.90)$ & ns \\
\hline Age group (years) & $t$-test & LMT-Simple & & WMp & & LMT-Complex & $t$-test \\
\hline $7-8$ & -2.73 & $2.36(1.09)$ & $\neq$ & 3 & $=$ & $2.77(0.81)$ & $\mathrm{ns}$ \\
\hline $9-10$ & -2.55 & $3.16(1.65)$ & $\neq$ & 4 & $\neq$ & $3.24(0.93)$ & -4.11 \\
\hline $11-12$ & ns & $4.86(1.11)$ & $=$ & 5 & $=$ & $4.67(1.15)$ & ns \\
\hline $13-14$ & -4.67 & $4.73(1.28)$ & $\neq$ & 6 & $=$ & $5.32(1.62)$ & ns \\
\hline $15-16$ & -5.56 & $5.96(0.93)$ & $\neq$ & 7 & $\neq$ & $6.48(1.19)$ & -2.18 \\
\hline $18+$ & -5.20 & $6.31(0.68)$ & $\neq$ & 7 & $=$ & $6.73(1.54)$ & ns \\
\hline
\end{tabular}

Note. $p=0.05$. Equivalence tests were computed using one sample $t$-tests. WMp: predicted WM, =: equivalent, and $\neq$ : not equivalent (SD).

observed in the LMT-Simple in early childhood, although further research on the effect of presentation modality on active rehearsal strategies is warranted. Despite the rationale for performance differences across visuospatial and verbal WM tasks, similar developmental trajectories in CMT- and LMT-Complex suggest that these discrepancies are reflective of differences in task-specific maintenance strategies more than neurodevelopmental processes underlying verbal and nonverbal WM.

4.2. Interference and Task Complexity. While mean performance scores across tasks varied with content domain, verbal and visuospatial developmental trajectories across age remained relatively invariant and instead differed based on task complexity. Our results demonstrate that the rate at which WM develops hinges upon the degree to which a task requires executive processes. In particular, participants showed greater consistency in performance on visuospatial and verbal WM tasks that included higher levels of interference and concurrent inhibitory control (e.g., CMT- and LMT-Complex) than simple tasks (CMT- and LMT-Simple) requiring only maintenance and recall of task items.

In contrast to the systematic growth patterns observed in the complex CMT and LMT, the developmental change in WM in CMT-Simple during early childhood was dramatic. Similar to short term memory models of WM, which show steep increases in memory span occurring between four and eight years and gradual increases up to about 12 years of age [9], performance scores in CMT-Simple markedly increased between seven and 12 years, stabilizing in early adolescence and improving only slightly during late adolescence and young adulthood. These findings imply that the addition of interference, at least within the visuospatial domain, increased the age at which optimal performance was reached.

The LMT-Simple did not perform as expected. Comparatively lower estimates of WM growth, especially later in development, when adolescents (aged 13 to 16 years) underperform relative to their performance on the more difficult LMT-Complex, may reflect differences in task design, namely, the level of sustained attention required to extract taskrelevant items. The layout of task items may not facilitate effective chunking strategies, whose process task analysis (see Appendix A) is taken into account in the computation of task WM demand. As such, the graphemic features in LMT-Simple may need to be modified in future studies investigating developmental changes on this task.

\section{Conclusions}

Our findings, supported through a strong equivalence of CMT and LMT complex to developmental predictions, demonstrate that controlled effortful attention, mediated through interference, is an important source of cognitive variance underlying developmental growth in WM capacity. Although performance trajectories were similar for complex verbal and visuospatial WM tasks and were predicted by age, the underlying mechanisms for processing verbal and nonverbal material appear to be qualitatively different. In contrast, the age-related patterns for the two simple tasks were more variable. In accordance with an inhibition view of WM which suggests that individual differences in WM capacity are only evident in complex tasks containing proactive interference [35], our findings suggest that developmental growth of WM capacity is primarily driven by a domain general executive that maintains tasks goals and resists distraction. Future work should examine the neural correlates and behavioural manifestations of verbal and visuospatial WM to elucidate the different brain mechanisms underlying these cognitive processes.

\section{Conflict of Interests}

The authors declare that there is no conflict of interests regarding the publication of this paper.

\section{Acknowledgment}

This work was supported by a grant from the Natural Sciences and Engineering Research Council (NSERC 138502-09). 


\section{References}

[1] N. Cowan and T. P. Alloway, "Development of memory in childhood," in The Development of Memory in Infancy and Childhood, M. L. Courage and N. Cowan, Eds., pp. 303-342, Psychological Press, Hove, UK, 2009.

[2] A. Baddeley, "Working memory and language: an overview," Journal of Communication Disorders, vol. 36, no. 3, pp. 189-208, 2003.

[3] N. Garon, S. E. Bryson, and I. M. Smith, "Executive function in preschoolers: a review using an integrative framework," Psychological Bulletin, vol. 134, no. 1, pp. 31-60, 2008.

[4] A. Miyake, N. P. Friedman, M. J. Emerson, A. H. Witzki, A. Howerter, and T. D. Wager, "The unity and diversity of executive functions and their contributions to complex "frontal lobe" tasks: a latent variable analysis," Cognitive Psychology, vol. 41, no. 1, pp. 49-100, 2000.

[5] A. R. A. Conway and K. Kovacs, "Individual differences in intelligence and working memory: a review of latent variable models," in The Psychology of Learning and Motivation, B. H. Ross, Ed., pp. 233-270, Elsevier, New York, NY, USA, 2013.

[6] V. R. Simmering and S. Perone, "Working memory capacity as a dynamic process," Frontiers in Psychology, vol. 3, article 567, 2012.

[7] M. Arsalidou, "Working memory capacity: the need for process task-analysis," Frontiers in Psychology, vol. 4, no. 257, Article ID Article 257, 2013.

[8] T. P. Alloway, S. E. Gathercole, and S. J. Pickering, "Verbal and visuospatial short-term and working memory in children: are they separable?" Child Development, vol. 77, no. 6, pp. 1698-1716, 2006.

[9] M. Luciana and C. A. Nelson, "Assessment of neuropsychological function through use of the Cambridge Neuropsychological Testing Automated Battery: performance in 4- to 12-year-old children," Developmental Neuropsychology, vol. 22, no. 3, pp. 595-624, 2002.

[10] G. Koppenol-Gonzalez, S. Bouwmeester, and J. Vermunt, "The development of verbal and visual working memory processes: a latent variable approach," Journal of Experimental Child Psychology, vol. 111, no. 3, pp. 439-454, 2012.

[11] J. N. Giedd, "Structural magnetic resonance imaging of the adolescent brain," Annals of the New York Academy of Sciences, vol. 1021, pp. 77-85, 2004.

[12] J. R. Best and P. H. Miller, "A developmental perspective on executive function," Child Development, vol. 81, no. 6, pp. 16411660, 2010.

[13] M. Arsalidou, J. Pascual-Leone, and J. Johnson, "Misleading cues improve developmental assessment of working memory capacity: the color matching tasks," Cognitive Development, vol. 25, no. 3, pp. 262-277, 2010.

[14] J. Pascual-Leone, "A mathematical model for the transition rule in Piaget's developmental stages," Acta Psychologica, vol. 32, pp. 301-345, 1970.

[15] J. Pascual-Leone and J. Johnson, "A dialectical constructivist view of developmental intelligence," in Handbook of Understanding and Measuring Intelligence, O. Wilhelm and R. W. Engle, Eds., pp. 161-200, Sage, London, UK, 2005.

[16] G. S. Halford, N. Cowan, and G. Andrews, "Separating cognitive capacity from knowledge: a new hypothesis," Trends in Cognitive Sciences, vol. 11, no. 6, pp. 236-242, 2007.
[17] N. Cowan, "The magical number 4 in short-term memory: a reconsideration of mental storage capacity," Behavioral and Brain Sciences, vol. 24, no. 1, pp. 87-114, 2001.

[18] G. A. Miller, "The magical number seven, plus or minus two: some limits on our capacity for processing information," Psychological Review, vol. 63, no. 2, pp. 81-97, 1956.

[19] N. Cowan, "The magical mystery four: how is working memory capacity limited, and why?" Current Directions in Psychological Science, vol. 19, no. 1, pp. 51-57, 2010.

[20] J. Pascual-Leone and J. Johnson, "The psychological unit and its role in task analysis: a reinterpretation of object permanence," in Criteria for Competence: Controversies in the Conceptualization and Assessment of Children's Abilities, M. Chandler and M. Chapman, Eds., pp. 153-187, Erlbaum, Hillsdale, NJ, USA, 1991.

[21] S. Morra, "Issues in working memory measurement: testing for M capacity," International Journal of Behavioral Development, vol. 17, no. 1, pp. 143-159, 1994.

[22] J. Pascual-Leone and J. Johnson, "A developmental theory of mental attention," in Cognitive Development and Working Memory: From Neo-Piagetian to Cognitive Approaches, P. Barrouillet and and V. Gaillard, Eds., pp. 13-46, New York, NY, USA, 2011.

[23] J. Johnson, S. J. Howard, and J. Pascual-Leone, "Attentional processes subserving working memory: mental versus perceptual attention," Unpublished manuscript, York University, Toronto, ON, Canada, 2011.

[24] R. Miller, J. Pascual-Leone, C. Campbell, and T. Juckes, "Crosscultural similarities and differences on two neo-Piagetian cognitive tasks," International Journal of Psychology, vol. 24, no. 3, pp. 293-313, 1989.

[25] T. Globerson, "Mental capacity and cognitive functioning: developmental and social class differences," Developmental Psychology, vol. 19, no. 2, pp. 225-230, 1983.

[26] J. Pascual-Leone, "Can we model organismic causes of working memory, efficiency and fluid intelligence? A meta-subjective perspective," Intelligence, vol. 41, no. 5, pp. 738-743, 2013.

[27] J. Pascual-Leone, "Organismic processes for neo-Piagetian theories: a dialectical causal account of cognitive development," International Journal of Psychology, vol. 22, no. 5-6, pp. 531-570, 1987.

[28] J. Pascual-Leone, "Learning and development as dialectical factor in cognitive growth," Human Development, vol. 38, no. 6, pp. 338-348, 1995.

[29] N. Cowan, E. M. Elliott, S. J. Saults et al., "On the capacity of attention: its estimation and its role in working memory and cognitive aptitudes," Cognitive Psychology, vol. 51, no. 1, pp. 42100, 2005.

[30] R. W. Engle, M. J. Kane, and S. W. Tuholski, "Individual differences in working memory capacity and what they tell us about controlled attention, general fluid intelligence, and functions of the prefrontal cortex," in Models of Working Memory: Mechanisms of Active Maintenance and Executive Control, A. Miyake and P. Shah, Eds., pp. 102-134, Cambridge University Press, New York, NY, USA, 1999.

[31] H. M. Conklin, M. Luciana, C. J. Hooper, and R. S. Yarger, "Working memory performance in typically developing children and adolescents: behavioral evidence of protracted frontal lobe development," Developmental Neuropsychology, vol. 31, no. 1, pp. 103-128, 2007.

[32] M. D'Esposito, B. R. Postle, D. Ballard, and J. Lease, "Maintenance versus manipulation of information held in working memory: an event-related fMRI study," Brain and Cognition, vol. 41, no. 1, pp. 66-86, 1999. 
[33] M. Arsalidou, J. Pascual-Leone, J. Johnson, D. Morris, and M. J. Taylor, "A balancing act of the brain: activations and deactivations driven by cognitive load," Brain and Behavior, vol. 3, no. 3, pp. 273-285, 2013.

[34] S. E. Gathercole and G. J. Hitch, "Developmental changes in short-term memory: a revised working memory perspective," in Theories of Memory, A. Collins, S. E. Gathercole, M. A. Conway, and P. E. Morris, Eds., pp. 189-210, Elbaum, Hove, UK, 1993.

[35] E. S. Darowski, E. Helder, R. T. Zacks, L. Hasher, and D. Z. Hambrick, "Age-related differences in cognition: the role of distraction control," Neuropsychology, vol. 22, no. 5, pp. 638644, 2008.

[36] J. Pascual-Leone, J. Johnson, S. Baskind, S. Dworsky, and E. Severtson, "Culture-fair assessment and the processes of mental attention," in Experience of Mediated Learning: An Impact of Feuerstein's Theory in Education and Psychology, A. Kozulin and and Y. Rand, Eds., pp. 191-214, Emerald Group, Bingley, UK, 2000.

[37] M. Petrides, B. Alivisatos, E. Meyer, and A. C. Evans, "Functional activation of the human frontal cortex during the performance of verbal working memory tasks," Proceedings of the National Academy of Sciences of the United States of America, vol. 90, no. 3, pp. 878-882, 1993.

[38] V. M. Vogan, B. R. Morgan, W. Lee, T. L. Powell, M. L. Smith, and M. J. Taylor, "The neural correlates of visuo-spatial working memory in children with autism spectrum disorder: effects of cognitive load," Journal of Neurodevelopmental Disorders, vol. 6 , no. 1, pp. 1-15, 2014.

[39] C. Rottschy, R. Langner, I. Dogan et al., "Modelling neural correlates of working memory: a coordinate-based meta-analysis," NeuroImage, vol. 60, no. 1, pp. 830-846, 2012.

[40] S. J. Pickering, "The development of visuo-spatial working memory," Memory, vol. 9, no. 4-6, pp. 423-432, 2001.

[41] G. Ben-Yehudah and J. A. Fiez, "Development of verbal working memory," in Human Behavior, Learning, and the Developing Brain: Typical Development, D. Coch, K. W. Fischer, and G. Dawson, Eds., pp. 301-328, Guilford Press, New York, NY, USA, 2007.

[42] W. Adams and D. Sheslow, WRAML: Wide Range Assessment of Memory and Language, Jastak Assessment Systems, Wilmington, Del, USA, 1990.

[43] G. J. Hitch, S. Halliday, A. M. Schaafstal, and J. M. C. Schraagen, "Visual working memory in young children," Memory \& Cognition, vol. 16, no. 2, pp. 120-132, 1988.

[44] N. Cowan, "The differential maturation of two processing rates related to digit span," Journal of Experimental Child Psychology, vol. 72, no. 3, pp. 193-209, 1999. 

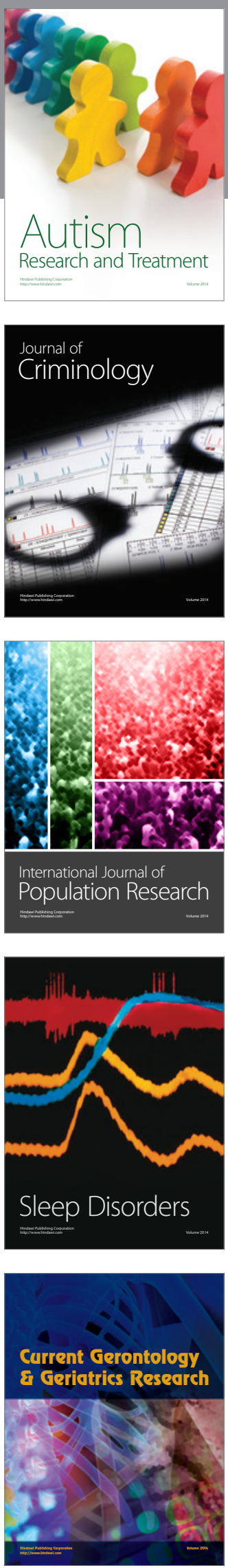
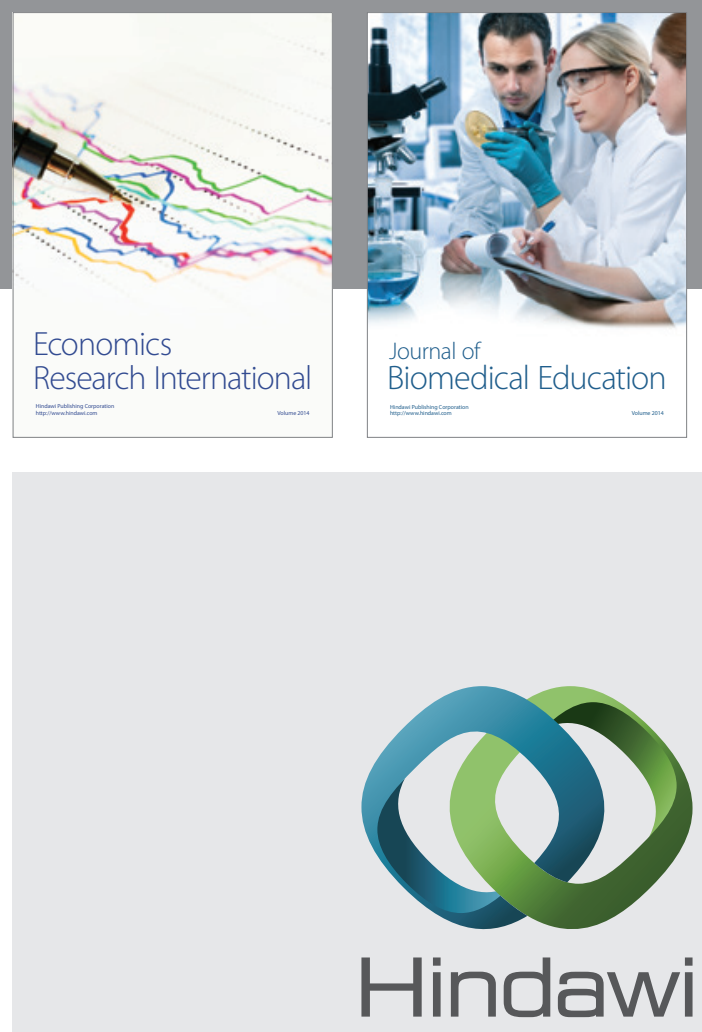

Submit your manuscripts at

http://www.hindawi.com
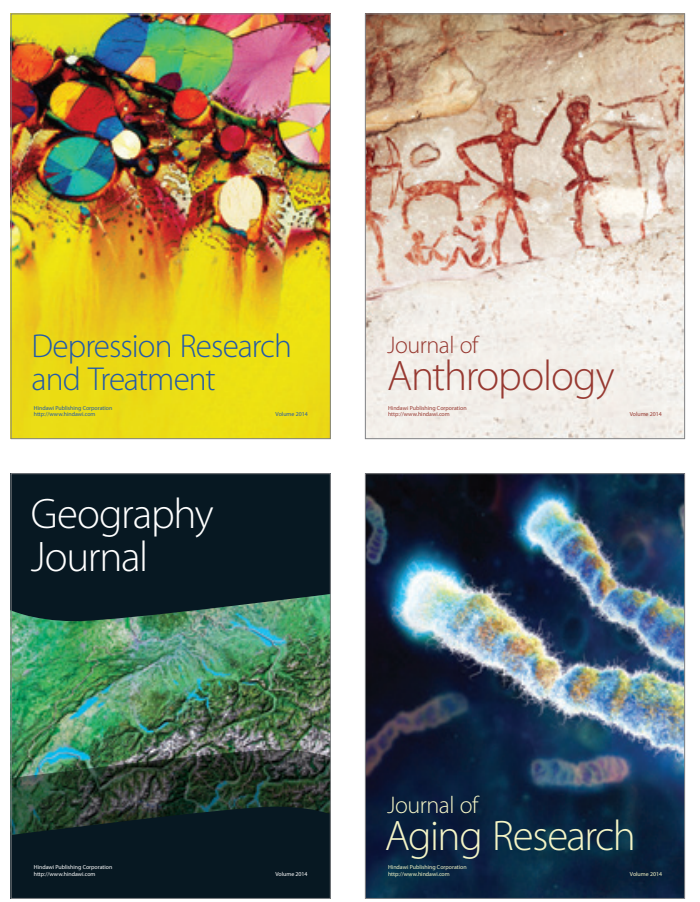
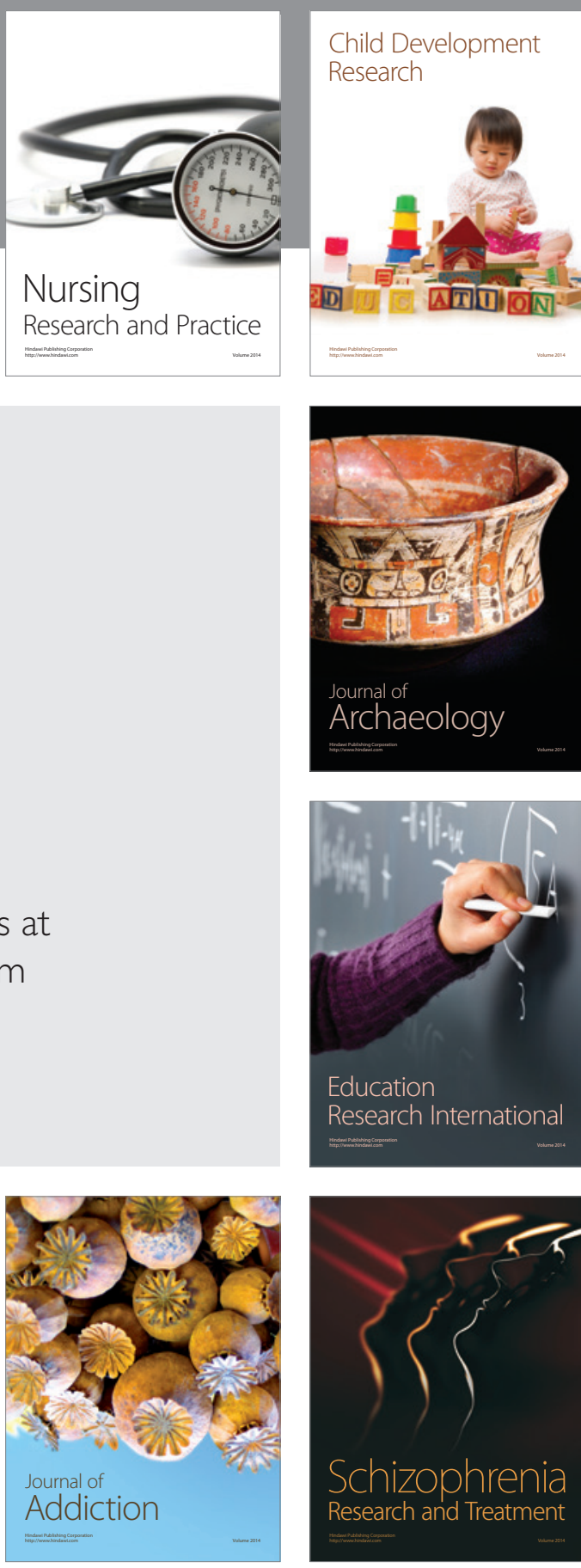

(D)
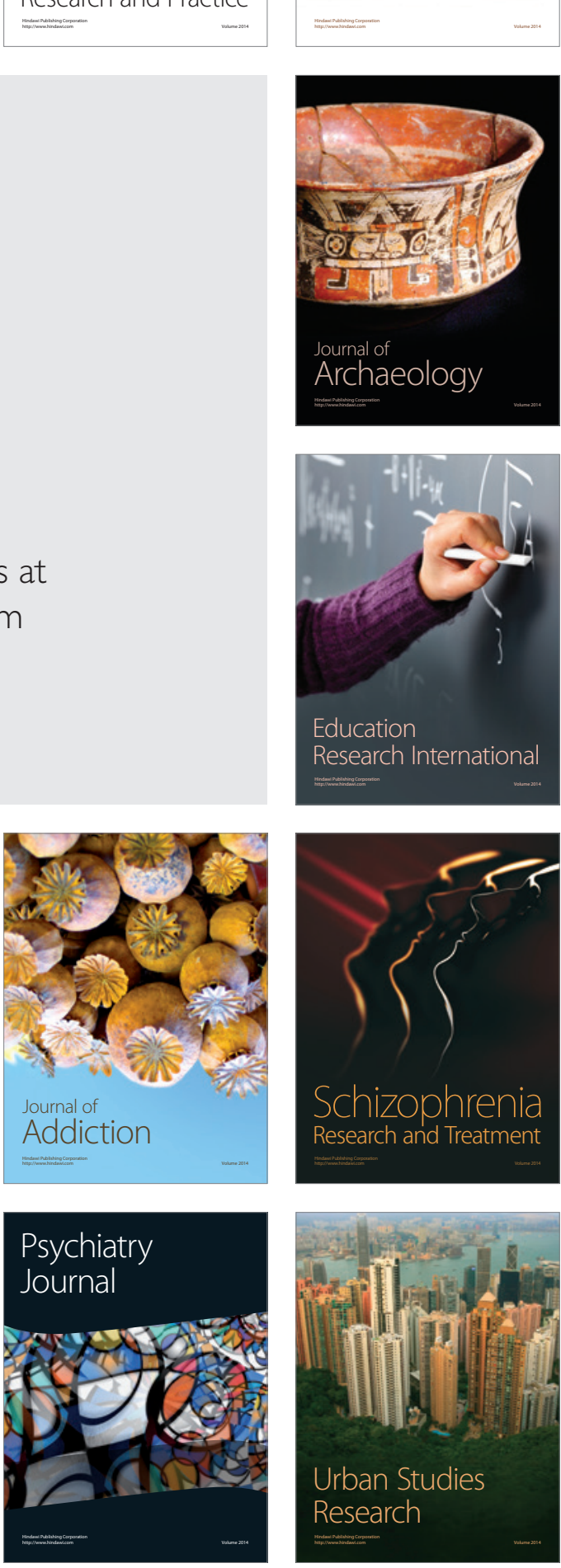